\title{
Cyclic AMP is a key regulator of M1 to M2a phenotypic conversion of microglia in the presence of Th2 cytokines
}

\author{
Mousumi Ghosh ${ }^{1,2^{*}}$, Yong Xu $\mathrm{Xu}^{1}$ and Damien D. Pearse ${ }^{1,2,34^{*}}$
}

\begin{abstract}
Background: Microglia and macrophages play a central role in neuroinflammation. Pro-inflammatory cytokines trigger their conversion to a classically activated (M1) phenotype, sustaining inflammation and producing a cytotoxic environment. Conversely, anti-inflammatory cytokines polarize the cells towards an alternatively activated (M2), tissue reparative phenotype. Elucidation of the signal transduction pathways involved in M1 to M2 phenotypic conversion may provide insight into how the innate immune response can be harnessed during distinct phases of disease or injury to mediate neuroprotection and neurorepair.

Methods: Microglial cells (cell line and primary) were subjected to combined cyclic adenosine monophosphate (cyclic AMP) and IL-4, or either alone, in the presence of pro-inflammatory mediators, lipopolysaccharide (LPS), or tumor necrosis factor-a (TNF-a). Their effects on the expression of characteristic markers for M1 and M2 microglia were assessed. Similarly, the M1 and M2 phenotypes of microglia and macrophages within the lesion site were then evaluated following a contusive spinal cord injury (SCI) to the thoracic (T8) spinal cord of rats and mice when the agents were administered systemically.
\end{abstract}

Results: It was demonstrated that cyclic AMP functions synergistically with IL-4 to promote M1 to M2 conversion of microglia in culture. The combination of cyclic AMP and IL-4, but neither alone, induced an $\mathrm{Arg}^{-1} \mathrm{1}^{+} / \mathrm{iNOS}^{-}$cell phenotype with concomitant expression of other M2-specific markers including TG2 and RELM-a. M2-converted microglia showed ameliorated production of pro-inflammatory cytokines (TNF-a and IP-10) and reactive oxygen species, with no alteration in phagocytic properties. M2a conversion required protein kinase A (PKA), but not the exchange protein directly activated by cyclic AMP (EPAC). Systemic delivery of cyclic AMP and IL-4 after experimental $\mathrm{SCl}$ also promoted a significant M1 to M2a phenotypic change in microglia and macrophage population dynamics in the lesion.

Conclusions: Using primary microglia, microglial cell lines, and experimental models of CNS injury, we demonstrate that cyclic AMP levels are a critical determinant in M1-M2 polarization. High levels of cyclic AMP promoted an Arg-1 ${ }^{+}$ M2a phenotype when microglia were activated with pro-inflammatory stimuli and Th2 cytokines. Th2 cytokines or cyclic AMP independently did not promote these changes. Phenotypic conversion of microglia provides a powerful new therapeutic approach for altering the balance of cytotoxic to reparative microglia in a diversity of neurological diseases and injury.

Keywords: Interleukin, Alternative activation, Repair, Inflammation, Innate immunity, M1, M2, Arginase, Inos, Phenotype

\footnotetext{
*Correspondence: mghosh@med.miami.edu; dpearse@med.miami.edu

${ }^{1}$ The Miami Project to Cure Paralysis, University of Miami Miller School of

Medicine, Miami, FL 33136, USA

Full list of author information is available at the end of the article
} 


\section{Background}

CNS resident microglia and infiltrating macrophages play a pivotal role in the acute inflammatory response to neurological injury and disease [1], altering the lesion environment to either a cytotoxic milieu characterized by ongoing tissue damage and cell death [2] or a reparative environment, defined by extracellular matrix remodeling, angiogenesis, and axonal regrowth [3]. The functions of these cells and their immunophenotypical characteristics are governed by a variety of signals produced both systemically and at the site of tissue injury, which drive them from a resting state to either a "classically activated," proinflammatory M1 phenotype or an "alternatively activated," reparative M2 phenotype [4]. Activators of the M1 phenotype, tumor necrosis factor- $\alpha$ (TNF- $\alpha$ ), and interferon- $\gamma$ (IFN- $\gamma$ ), induce microglia to produce proinflammatory cytokines, oxidative metabolites, and proteases [5], which although important for microbial defense [6], exacerbate the extent of the initial neural injury and associated dysfunction [7]. Markers of the M1 phenotype include inducible nitric oxide (iNOS), cyclo-oxygenase-2 (COX-2), and cell surface markers such as CD16, CD86, and MHC II. Conversely, the M2 phenotype results from microglial activation in the presence of anti-inflammatory cytokines, including interleukin (IL)-4, IL-10, IL-13, and tumor growth factor- $\beta$ (TGF- $\beta$ ) [8]. M2 microglia produce a variety of growth factors including vascular endothelial growth factor (VEGF), brain-derived neurotrophic factor (BDNF), and platelet-derived growth factor (PDGF), which are important for promoting angiogenesis, oligodendrocyte maturation, axonal regrowth, and remyelination repair that is conducive to wound healing [9]. The M2a phenotype, a subset of the alternatively activated state of microglia or macrophages, is characterized by the markers Arginase-1 (Arg-1), transglutaminase-2, RELM- $\alpha$, and YM1 $[8,9]$.

Though studies have suggested that the presence of Th2 cytokines alone are sufficient for converting a proinflammatory, activated M1 form to an M2a phenotype, a persistent M1-M2a conversion using this approach has not been achievable in vivo; macrophages that have been stimulated towards this alternatively activated phenotype by Th2 cytokines alone are unable to retain their immunophenotypical and functional properties when placed within the injured or diseased CNS milieu, reverting instead to the M1 phenotype [10]. In the current study, we have demonstrated that cyclic adenosine monophosphate (cyclic AMP) levels are a critical determinant in pronounced M1 to M2a phenotype conversion when microglial cells are in the presence of proand anti-inflammatory stimuli. The elevation of cyclic AMP in microglia or macrophages, through the use of adenylyl cyclase (AC) activators, phosphodiesterase (PDE) inhibitors, synthetic cyclic AMP analogs, or $\beta$ - adrenergic receptor agonists, inhibits the production of pro-inflammatory genes [11] that are controlled by the master immune regulator, transcription factor nuclear factor kappa B (NF-kB; [12]). We suggest that the role of cyclic AMP signaling is to suppress the M1 phenotype, largely by inhibiting NF- $\mathrm{kB}$, thus potentiating the alternative activation of microglia into an augmented, M2a phenotypic state synergistically in the presence of Th2 cytokines.

\section{Methods \\ Reagents}

Cytokines were purchased from PeproTech (Rocky Hills, NJ). Cyclic AMP analogs were obtained from the BIOLOG Life Science Institute (Bremen, Germany) except dioctynyl cyclic AMP, which was obtained from Santa Cruz Biotechnology Inc. (Dallas, TX). Lipopolysaccharide (LPS) was purchased from Sigma-Aldrich (St. Louis, MO).

\section{BV2 microglial cell culture}

The immortalized BV2 microglial cell line employed for in vitro experiments was derived from the C57BL/6 mouse [13] and exhibits phenotypic and functional properties of primary microglia [14]. BV2 microglia were cultured at $37{ }^{\circ} \mathrm{C}, 5 \% \mathrm{CO}_{2}$ in Dulbecco's modified Eagle's medium (DMEM; Gibco, Life Technologies Corporation, Grand Island, NY) supplemented with $10 \%$ heatinactivated fetal bovine serum (FBS; HyClone, Logan, UT) and 100 units $/ \mathrm{mL}$ each of penicillin and streptomycin (Sigma-Aldrich). Prior to experimental use, BV2 microglia were seeded on either six-well culture plates $\left(5 \times 10^{4}\right.$ cells/ well for biochemistry experiments) or eight-well chamber slides $\left(5 \times 10^{3}\right.$ cells/well for immunocytochemistry) and grown to $70-80 \%$ for experimental use. BV2 microglia were treated with various cytokines or pharmacological agents based on the experimental schema described below.

\section{Primary microglial cell culture}

Primary cultures of microglia were prepared from the cerebral cortex of neonatal (P1) wild type C57Bl/6J mice (JAX ${ }^{\odot}$ Mice and Services, Bar Harbor, ME) using previously published methods [15]. Briefly, the cerebral cortices were dissected and cut into $2-\mathrm{mm}$ small pieces. Tissue was suspended in cold DMEM (Gibco, Life Technologies Corporation) and triturated using a P1000 plastic tip. The resulting cell suspension was passed through a $100-\mu \mathrm{m}$ cell strainer and then centrifuged at $\times 1000 \mathrm{rpm}$ for $10 \mathrm{~min}$. The supernatant was removed and the pellet re-suspended in DMEM containing $10 \%$ FBS and $10 \%$ horse serum (Gibco, Life Technologies Corporation). The cell suspension was plated on polylysine (PLL)-coated T75 flasks and 
incubated at $37{ }^{\circ} \mathrm{C}$ for 7 to 10 days. Culture flasks were then placed on an orbital shaker at X230 rpm for $3 \mathrm{~h}$, and the media, containing detached microglia, was harvested. Following centrifugation for $10 \mathrm{~min}$ at $\times 1000 \mathrm{rpm}$, a pellet containing the microglial cell fraction was acquired and re-suspended in the same media as described above. Highly pure microglial cell cultures were obtained using this method ( $>95 \%$ as assessed using immunocytochemistry for CD11b (AbD Serotec; Raleigh, NC) and iba1 (Wako Pure Chemicals; Richmond, VA)). Primary microglia were diluted to the desired cell concentration and plated for at least $24 \mathrm{~h}$ before experimental use.

\section{M1 to M2 phenotypic conversion}

M1 activation was achieved with LPS $(100 \mathrm{ng} / \mathrm{ml})$ or TNF- $\alpha$ (10 ng/mL). For M1 to M2a phenotype conversion, IL-4 $(10 \mathrm{ng} / \mathrm{mL})$ was used in combination with cyclic AMP analogs (dibutyryl cyclic AMP; $(1 \mathrm{mM}), 6$ Phe-cyclic AMP; $(100 \mu \mathrm{M})$, CPT-2'O cyclic AMP; $(100 \mu \mathrm{M}))$ beginning at 15 min prior to M1 activation. Cell supernatants were analyzed for cytokine levels, cell lysates prepared, or the cells fixed with $4 \%$ paraformaldehyde $24 \mathrm{~h}$ later to measure the expression of M1 and M2a phenotype markers. All data are representative of at least three independent experiments.

\section{Assessment of phagocytic function}

Phagocytic function was evaluated based on the uptake of phycoerythrin (PE)-conjugated latex beads (Phagocytosis Assay Kit; Cayman Chemical, Ann Arbor, MI) as per the manufacturer's protocol. Percent phagocytic cells was quantified in relation to the total number of Hoechstpositive nuclei in four random fields per treatment well and averaged across five independent experiments by fluorescence microscopy.

\section{Western blotting}

Immunoblotting was performed on culture supernatants, total cell lysates, or spinal cord (T7-9) tissue homogenates to quantify the expression of M1 and M2a markers according to previously published methods [15]. Nitrocellulose membranes (Bio-Rad Laboratories) were probed with specific primary antibodies; Arg-1 (1:2,000; GeneTex Inc., Irvine, CA), iNOS (1:1,000; Cell Signaling Technology, Boston, MA), TNF- $\alpha$ (1:500; Life Technologies Corp.), and $\beta$-actin (Sigma-Aldrich). The optical density of the bands (arbitrary units) was measured with an imaging densitometer (Bio-Rad Laboratories) and normalized to $\beta$-actin levels. The data represents values from three independent experiments.

\section{ELISA}

Levels of the pro-inflammatory cytokines in culture supernatants was quantified using mouse TNF- $\alpha$, IP-10, and IL-1 $\beta$ enzyme-linked immunosorbent assays (ELISA; Pepro Tech, Rocky Hill, NJ) according to the manufacturer's protocols. Values were obtained as picogram per milliliter of the culture supernatant and expressed as absolute values and compared between each of the treatment conditions. The data presented are the mean \pm SEM of four independent experiments.

\section{Determination of reactive oxygen species}

The presence of reactive oxygen species (ROS) was measured using the non-fluorescent dye $2^{\prime}, 7^{\prime}$-dichlorofluorescein diacetate (DCFH-DA; Molecular Probes, Eugene, OR) as per the manufacturer's instructions. DCF fluorescence measurements were made in 96-well plates using a SpectraMax 5 microplate reader at $485 \mathrm{~nm}$ for excitation and $530 \mathrm{~nm}$ for emission. The data obtained were representative of three independently conducted experiments.

\section{Nitrite assay}

Microglia nitrite production was assessed by measuring total nitrite concentration, a stable oxidation product of NO by the Griess reaction. Briefly, culture supernatants $(50 \mu \mathrm{l})$ were mixed with an equal volume of Griess reagent (Life Technologies Corp.) in 96-well plates for $10 \mathrm{~min}$ at room temperature in the dark, and the absorbance at $570 \mathrm{~nm}$ was determined using a SpectraMax 5 microplate reader. Sodium nitrite at concentrations of 0 to $100 \mu \mathrm{M}$ was used as a standard. Each value indicates the mean \pm SEM and is representative of results obtained from three independent experiments.

\section{Immunocytochemistry}

M1 and M2 marker expression in microglia was examined using immunocytochemistry (ICC) according to previously published methods [15]. Fixed cells were probed with primary antibodies for the M1 phenotype, iNOS (1:200; BD Transduction Laboratories; Franklin Lakes, NJ), and COX-2 (1:200; Thermo Scientific, Rockford, IL) or the M2 phenotype, Arg-1 (1:200; GeneTex, Irvine, CA), RELM $\alpha /$ Fizz1 (1:100; PeproTech, Rocky Hill, NJ), or transglutaminase-2 (1:100; Thermo Fischer Scientific). In addition, the microglial cell markers anti-iba1 (1:5,000; Wako Pure Chemical Industries, Ltd., Tokyo, Japan) and CD68/ED1 (Macrosialin; 1:200; AbD Serotec) were used. For visualization, the secondary antibodies, goat antimouse Alexa-594 or anti-rabbit Alexa-594 (1:200, Life Technologies Corp.) were used along with the nuclear marker Hoechst 33342 (1:1,000; Life Technologies Corp.). The morphology of the cells was demarcated by staining with Phalloidin-488 (1:100; Life Technologies Corp). Images were acquired by sequential scanning of the immunostained cells with an Olympus Fluorescence confocal microscope (Olympus, Fluoview FV 1000). For each treatment condition, three to four randomly selected fields 
were imaged per well. Images obtained were representative of three independently conducted experiments.

\section{Imaging and analysis}

Confocal images were acquired by sequential scanning of the immunostained cells with an Olympus Fluorescence Microscope (Olympus, FluoView FV1000) at laser lines based on the specific Alexa-fluor secondaries used. In Adobe Photoshop CS6 (Adobe Systems, San Jose, CA) fluorescent images had the same universal adjustments applied: brightness $(+50)$, contrast $(+30)$, and smart sharpen (1.0 pixels).

\section{Measurement of fluorescent intensity per cell}

The per cell immunostaining fluorescence intensity of specific markers was quantified using Image J software (http://imagej.nih.gov/ij/). For each treatment condition, randomly selected cells $[10,11]$ per well/plate from independent experiments were identified by Hoechstpositive nuclei and imaged. These images were converted to grayscale, and the average per pixel intensity of the signal per cell (arbitrary units (a.u.) was recorded after background subtraction. These measurements were averaged across the cells analyzed per plate and then among group replicates for comparison of signal change across treatments.

\section{Spinal cord contusion injury}

Two models of spinal cord injury (SCI) were used in this study to examine the effects of IL4 and db-cyclic AMP on microglia and macrophage phenotypes in rodents. The first model employed adult Lewis rats (female, 180$200 \mathrm{~g}, n=3$ ), the second C57BL/6 (female, $25 \mathrm{~g}$ ) mice (both from Charles River Laboratories International Inc., Wilmington, MA). Animals were housed according to NIH guidelines and The Guide for the Care and Use of Animals. All animal procedures were approved by the University of Miami Institutional Animal Care and Use Committee (IACUC). The rats were subjected to a moderate $(25.0 \mathrm{~mm})$ thoracic (T8) spinal cord contusion induced by the MASCIS weight drop device developed at New York University [16] as described previously [17]. For the mouse model ( $n=3$ per group) animals were subjected to a 50 kdyne moderate spinal cord contusion at thoracic (T8) level using the Infinite Horizons (IH) impactor as described previously [18]. Post-operative care was provided as described in Patel et al. [19].

\section{Administration of M2a converting agents}

With the Lewis rat model, IL-4 and db-cyclic AMP were delivered by intravenous (i.v.) administration through a jugular vein catheter prepared as described previously [20]. A mix of db-cyclic AMP (50 mg/kg) and IL-4 $(30 \mu \mathrm{g} / \mathrm{kg})$ in $100 \mu \mathrm{l}$ of sterile, deionized water was administered as a single bolus injection with a $0.5-\mathrm{ml}$ tuberculin syringe attached to the i.v. catheter over a period of $2 \mathrm{~min}$ within $15 \mathrm{~min}$ of $\mathrm{SCI}$, followed by a $250-\mu \mathrm{l}$ physiological saline flush. For the mouse model, the animals received daily, intraperitoneal injections $(100 \mu \mathrm{l})$ of the agents at the same concentrations beginning at 30 min post-SCI for 7 days until end-point.

\section{Animal perfusion and tissue extraction}

Animals were deeply anesthetized $(150 \mathrm{mg} / \mathrm{kg}$ ketamine, $10 \mathrm{mg} / \mathrm{kg}$ xylazine) and transcardially perfused and the extracted CNS tissue post-fixed and cryoprotected as described elsewhere [21]. The T7-9 spinal cord was cryosectioned at a thickness of $20 \mu \mathrm{m}$ (coronal) on a Leica CM3050S Cryostat (Leica Microsystems Inc., Buffalo Grove, IL). For biochemistry, animals were deeply anesthetized at $24 \mathrm{~h}$ post-SCI and decapitated. A spinal cord segment $(5 \mathrm{~mm})$ encompassing the injury epicenter dissected and snap frozen in liquid nitrogen for storage at $-80{ }^{\circ} \mathrm{C}$ until processed for analysis.

\section{Immunohistochemistry}

Coronal, $20-\mu \mathrm{m}$ cryosectioned sections of injured spinal cord tissue (400- $\mu \mathrm{m}$ intervals) were immunohistochemically (IHC) stained for specific markers using methods as described elsewhere [20]. For IHC, the Arg-1 antibody (1:200) was combined with anti-CD68 (ED1; 1:200; AbD Serotec), Alexa-594 tagged Isolectin-IB4 (1:100; Life Technologies Corp.) or anti-iba1 (1:5,000; Wako) along with appropriate AlexaFluor conjugated secondary antibodies (1:200; Life Technologies Corp.) and Hoechst 33342 (Sigma-Aldrich).

\section{Quantification of microglial cells at the lesion site}

For quantifying the conversion of microglia and macrophages to M2a, cells displaying double immunoreactivity for Arginase-1 (Alexa-488 secondary antibody) and Alexa-594-tagged Isolectin-IB4 were quantified from projection images obtained using a confocal microscope (Olympus, Fluoview 1000) at $\times 40$ magnification. These images were taken from within the injury epicenter, from the dorsal funiculus region of the spinal cord $(n=3$ mice per group of IL- 4 and db-cyclic AMP-treated and SCIonly controls). Images were converted to 8-bit gray scale separately for each marker using Image J software, and a threshold value was obtained. The relative percent of cells that were displaying the M2a phenotype were analyzed by measuring the fluorescent intensity of the number of cells showing Arginase-1 immunoreactivity and the total number of Isolectin-IB4 ${ }^{+}$cells between the treated and the untreated groups. 


\section{Statistics}

Significant differences between groups were ascertained by a one-way analysis of variance (ANOVA) or a $t$ test, followed by a Bonferroni post-hoc analysis using Graph Pad Prism 4.0 (Graphpad Software, La Jolla, CA). All data was analyzed at the $95 \%$ confidence interval. Graphed data was expressed as the mean \pm standard error of the mean (SEM) according to the number of replicates or independent experiments. Asterisks or hashes included on the graphs indicate statistical differences between the treatment and control condition(s) with significance indicated at $\left.{ }^{* * * *}\right|^{\# \# \#} p<0.001,\left.{ }^{* * *}\right|^{\# \#} p<0.01$, or ${ }^{*} /^{\#} p<0.05$.

\section{Results}

Cyclic AMP and IL-4 synergize to augment expression of M2a phenotypic markers in microglia induced to an M1 form by pro-inflammatory mediators

BV2 microglia exposed to a pro-inflammatory stimulus, LPS, showed M1 phenotype induction as evidenced by pronounced expression of the characteristic markers iNOS and COX-2 and an absence of the M2a markers, Arginase-1, transglutaminase-2, and RELM- $\alpha$ (Fig. 1ae). Concurrent treatment of LPS-stimulated BV2 microglia with IL4 and db-cyclic AMP but, neither independently (data not shown), antagonized M1 marker expression while dramatically augmenting an M2a phenotype as demonstrated by robust expression of M2a markers (Fig. 1f-j). M1 to M2a phenotype conversion in BV2 microglia was demonstrated immunocytochemically also with another pro-inflammatory mediator, TNF- $\alpha$, in which, compared to untreated (Fig. $1 \mathrm{k}-\mathrm{l}$ ) or TNF- $\alpha$ stimulated controls (Fig. 1m, n), combined IL-4 and dbcyclic AMP induced robust Arginase-1 production and the antagonism of iNOS expression (Fig. 1s, t). Only minor changes in Arginase-1 and iNOS were observed in TNF- $\alpha$-stimulated BV2 microglia treated with IL-4 (Fig. 1o-p) or db-cyclic AMP (Fig. 1q-r) independently at the doses employed. Similarly, modest expression of transglutaminase-2, and RELM- $\alpha$ were detected with IL4 treatment, but the presence of db-cyclic AMP dramatically potentiated their expression and the alternatively activated M2a phenotype (data not shown).

The necessity of cyclic AMP for robust M1 to M2 phenotype conversion was further demonstrated biochemically by immunoblotting for the prototypical M2 marker Arginase-1 (Fig. 1u, v). In unstimulated, resting microglia (M0 phenotype), exposure to IL-4 alone, but not db-cyclic AMP, elevated levels of Arginase-1, indicative of a transition to an M2 phenotype. However, as observed with LPS- or TNF- $\alpha$-induced M1 microglia, exposure of the resting $\mathrm{M} 0$ microglia to the combination of IL-4 and db-cyclic AMP showed a much more pronounced expression of Arginase-1 (3.6-fold higher, $p<$ $0.001)$. In M1 activated microglia exposed to either LPS or TNF- $\alpha$, IL-4 and db-cAMP when given independently at the dose used did not induce robust Arginase-1 compared to concurrent application of IL-4 and db-cyclic AMP (Fig. 1u, v).

To examine the dose-dependent effects of IL-4 and db-cyclic AMP on the phenotype conversion of M1 microglia $\left(\mathrm{iNOS}^{+}\right.$) to M2 phenotype (Arginase- ${ }^{+}$), immunoblotting studies with titrated concentrations of these agents were performed. When the concentration of db-cyclic AMP was kept constant at $1 \mathrm{mM}$, IL-4 concentrations at and above $10 \mathrm{ng} / \mathrm{mL}$ were required to produce a significant induction of Arginase-1 expression and concomitant reduction in iNOS expression in LPSstimulated BV2 microglia (Fig. 1w, x). Similarly, maintaining IL-4 at $10 \mathrm{ng} / \mathrm{mL}$ demonstrated that db-cyclic AMP concentrations at and beyond $0.1 \mathrm{mM}$ could trigger a significant induction of Arginase-1 along with the parallel inhibition of iNOS expression during the phenotype conversion of M1 microglia to M2 (Fig. 1y, z).

\section{Cyclic AMP profoundly modifies cytokine secretion in} microglia stimulated to the M1 phenotype by LPS or TNF-a The cytokine secretory profile of untreated or resting (M0), M1 (induced by LPS or TNF- $\alpha$ stimulation), or M2a (converted from M1 with IL-4 and db-cyclic AMP) BV2 microglia was investigated in culture supernatants at $24 \mathrm{~h}$ using specific ELISAs for TNF- $\alpha$, IL-1 $\beta$, or IP-10. ELISAs for each of these major pro-inflammatory cytokines were performed from three independent experiments. Compared to M0, the LPS-treated form showed a significant increase in the levels of TNF- $\alpha$ (TNF- $\alpha$, 7.4-fold increase, $\mathrm{p}<0.001$; Fig. 2a). Similarly, TNF- $\alpha$ stimulation of the cells produced elevated concentrations of IP-10 in the culture supernatant at $24 \mathrm{~h}$ following treatment (IP-10, 4.9fold increase, $p<0.001$; Fig. 2b). IL-4 addition alone produced a modest, though significant reduction in the levels of TNF- $\alpha$ and IP-10 compared to stimulated controls; however, a major abrogation in the secretion of these cytokines was achieved with dbcyclic AMP or its combination with IL-4 (Fig. 2a, b). In contrast, IL-1 $\beta$ production following a 24-h TNF- $\alpha$ stimulus did not show any significant differences between the M0, M1, or M2a phenotypes, though M1 microglia exposed to db-cyclic AMP alone did show significantly enhanced levels of IL-1 $\beta$ (1.7-fold increase, $p<0.05)$ compared to stimulated controls (Fig. 2c).

\section{$M 1$ to $M 2$ conversion of microglia by the synergistic action of IL-4 and cyclic AMP can be observed at the single cell level}

The synergistic effects of cyclic AMP and IL-4 in regulating expression of Arginase-1 and iNOS was examined within single cells of primary cortical microglia challenged 


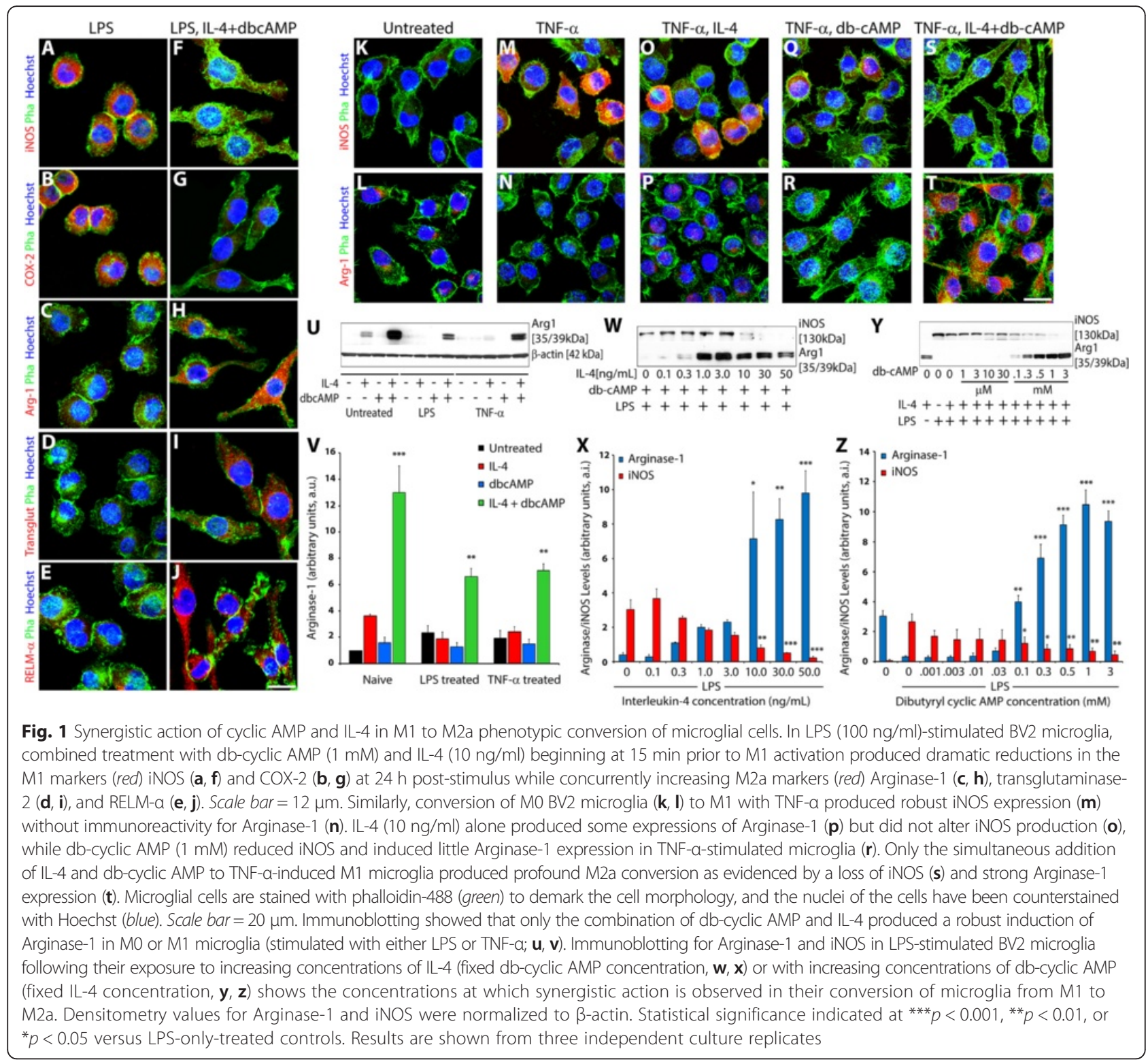

with TNF- $\alpha$ (Fig. $2 \mathrm{~d}-\mathrm{q}$ ). While levels of Arginase-1 and iNOS were low in M0 microglia and not significantly different from one another (Fig. $2 \mathrm{~d}-\mathrm{f}$ ), upon TNF- $\alpha$ stimulation, a profound increase in iNOS over Arginase- 1 was observed in single microglial cells (Fig. 2g-i). The exposure of M1 microglia to either IL-4 (Fig. 2j-l) or db-cyclic AMP (Fig. 2m-o) was not able to reverse the balance of Arginase- 1 to iNOS expression that significantly favored the later in single cells. In contrast, the concurrent exposure of M1 microglia to IL-4 and db-cyclic AMP switched the balance of Arginase-1 to iNOS expression to significantly favor Arginase-1 (Fig. 2p-r), indicative of an M1 to M2 phenotype conversion.
The cyclic AMP-protein kinase A pathway confers M1 to Arginase- $1^{+} \mathrm{M} 2 \mathrm{a}$ phenotype conversion in microglia The main effector pathways downstream of cyclic AMP are coordinated through PKA and the exchange protein directly activated by cyclic AMP (EPAC), which have been shown to regulate often distinct cellular processes in neural cells [22]. To probe the involvement of PKA and EPAC in M2a phenotype conversion, selective cyclic AMP analogs that activate PKA or EPAC were employed in combination with IL-4 in cultures of primary cortical microglial cells. Similar to db-cyclic AMP, the PKAspecific cyclic AMP analogs, dioctanoyl-cyclic AMP, and 6-phenyl-cyclic AMP, but not the EPAC-selective 


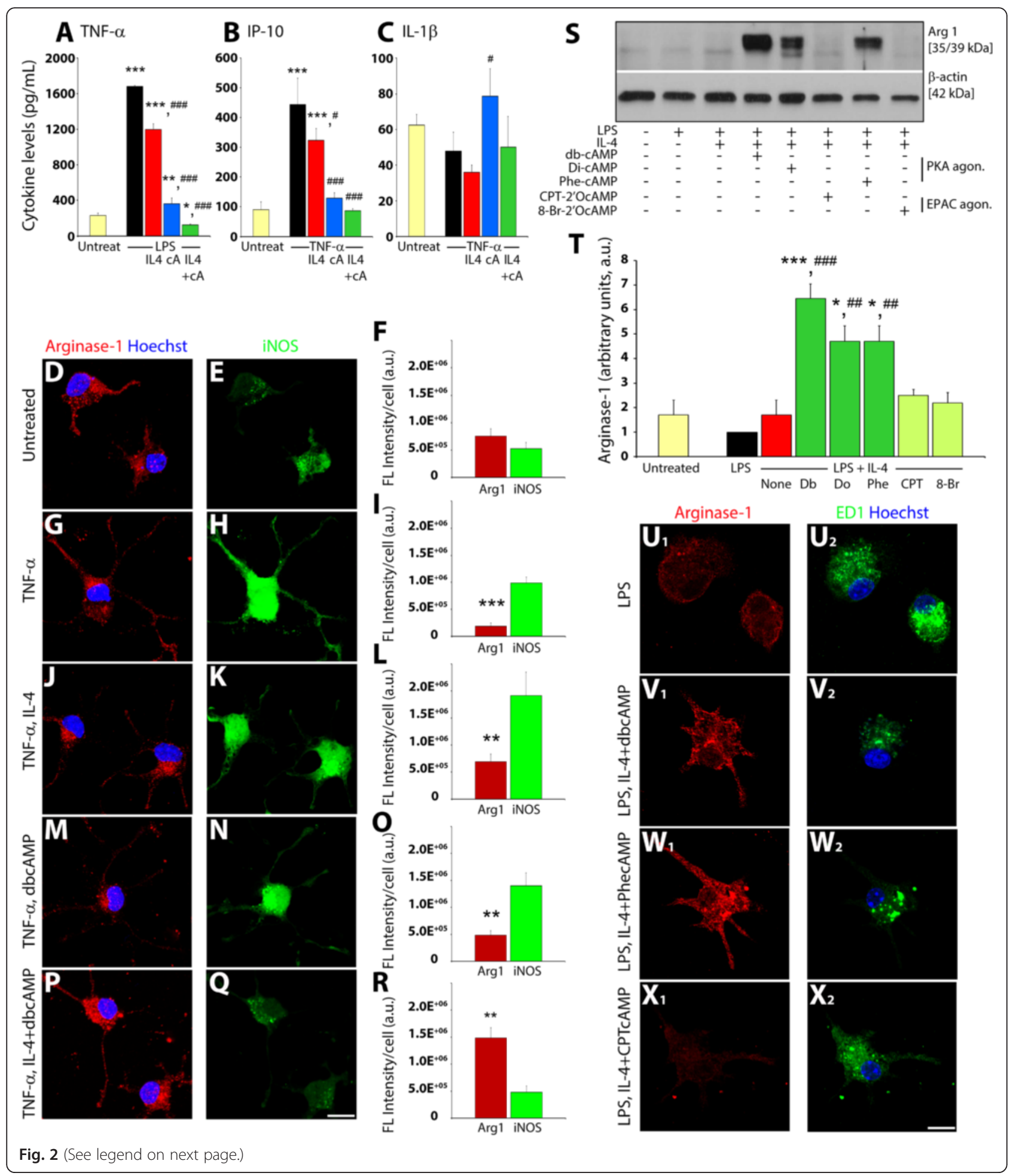




\begin{abstract}
(See figure on previous page.)
Fig. $2 \mathrm{M} 1$ to M2a conversion of microglia alters secretory profiles, occurs at the single cell level, and requires PKA, but not EPAC, signaling in the presence of IL-4. Treatment of M1 (LPS; $100 \mathrm{ng} / \mathrm{ml}$ or TNF-a stimulated $10 \mathrm{ng} / \mathrm{ml}$ ) BV2 microglia with db-cyclic AMP (1 mM) and IL-4 (10 ng/ml) beginning at $15 \mathrm{~min}$ prior to activation produced maximal abrogation of cytokines, TNF-a (a) and IP-10 (b) at $24 \mathrm{~h}$ post-stimulus. Levels of IL-1 $\beta$ remained statistically indifferent between M0, M1, and M2a phenotypes (c), with only db-cyclic AMP alone producing a significant increase over TNF-a stimulated controls. Examination of primary cortical microglia at the single cell level showed, compared to untreated M0 microglia (d, e), a significant shift in the ratio of Arginase-1 (red) to iNOS (green) expression, favoring the latter $(\mathbf{g}-\mathbf{i})$, occurred when the microglia were induced to the M1 form with TNF-a. The exposure of M1 microglia to either IL-4 (j-I) or db-cyclic AMP ( $\mathbf{m}-\mathbf{0}$ ) independently failed to alter this ratio. In contrast, concurrent use of db-cyclic AMP and IL-4 significantly shifted the ratio of Arginase-1 to iNOS expression to one favoring the former (p-r), indicative of M1 to M2a phenotypic conversion. Statistical significance indicated at ${ }^{* * *} p<0.001$ or ${ }^{* *} p<0.01$ versus iNOS FL intensity/cell. Scale bar $=15 \mu \mathrm{m}$. $\mathbf{s}$ Immunoblotting for Arginase-1 in BV2 microglia showed little expression in MOuntreated cells or M1 cells induced with LPS and either untreated or exposed to IL-4. Concurrent delivery of IL-4 with cyclic AMP analogs showed that those that selectively activated PKA (db-cyclic AMP, 1 mM; dioctanoyl-cyclic AMP, $100 \mu \mathrm{M}$; phenyl-cyclic AMP, $100 \mu M$ ), but not EPAC (CPT-2'O methyl-cyclic AMP, $100 \mu \mathrm{M}$ and 8-bromo-2'O methyl-cyclic AMP, $100 \mu \mathrm{M}$ ), induced robust Arginase-1 expression, indicative of M1 to M2a phenotypic conversion. $\mathbf{t}$ Densitometry values for Arginase-1 in untreated and treatment groups normalized to $\beta$-actin. The expression of Arginase-1 (red) and iNOS (green) in primary microglia treated with LPS alone $\left(\mathrm{u}_{1}-\mathrm{u}_{2}\right)$ or in conjunction with IL-4 and cyclic AMP analogs that activate PKA and/or EPAC $\left(\mathrm{V}_{1}-\mathrm{X}_{2}\right)$, shows the importance of PKA in mediating conversion towards an Arg1+/iNOS- phenotype in the presence of IL-4. Statistical significance indicated at ${ }^{* * *} p<0.001$ or ${ }^{*} p<0.05$ versus untreated controls or ${ }^{\# \# \#} p<0.001$ or ${ }^{\#} p<0.05$ versus LPS-only-treated controls. $\mathbf{c}-\mathbf{j}$ Immunocytochemical staining of primary microglia for Arginase-1 (red) and macrosialin (ED1; green) in control and treated microglia showed robust induction of Arginase-1 with PKA-selective, but not EPAC-selective, cyclic AMP analogs in the presence of LPS and IL-4. Cell nuclei were counterstained with Hoechst (blue). Scale bar $=15 \mu \mathrm{m}$. Results shown from three independent culture replicates
\end{abstract}

analogs, CPT-2'O methyl-cyclic AMP or 8-bromo-2'O methyl-cyclic AMP, induced a significant increase in Arginase-1 expression when combined with IL-4 treatment in M1 microglia (Fig. 2s-t). These results were then confirmed using immunocytochemistry for Arginase-1 in primary cultures of microglia (Fig. $2 \mathrm{u}-\mathrm{x}$ ). LPS-stimulated primary microglia exhibited little immunoreactivity for Arginase- 1 and robust expression of macrosialin (ED-1), a characteristic marker of activated microglia and macrophages (Fig. $2 u_{1}-u_{2}$ ). Strong Arginase- 1 immunoreactivity was observed in M1 microglia exposed to IL-4 when used in combination with the PKA activators db-cyclic AMP (Fig. $2 \mathrm{v}_{1}-\mathrm{v}_{2}$ ) and phenyl-cyclic AMP (Fig. $2 \mathrm{w}_{1}-\mathrm{w}_{2}$ ), but not with the EPAC-selective cyclic AMP analog CPT-2'O methyl-cyclic AMP (Fig. $2 \mathrm{x}_{1}-\mathrm{x}_{2}$ ).

\section{Cyclic AMP restores IL-4 mediated deficits of phagocytic activity in the M2a phenotype}

The phagocytic ability of M2a converted microglia following treatment with LPS and IL4, with or without dbcyclic AMP, was comparatively examined to LPSstimulated alone (M1) and M0 (no treatment) microglia through quantification of their capacity to phagocytose PE-conjugated latex beads. Compared to untreated cells (Fig. 3a), LPS stimulation of BV2 microglia to an M1 phenotype enhanced their ability to phagocytose PEconjugated beads (3.3-fold higher than untreated, $p<$ 0.001; Fig. 3b, f). In contrast, IL-4 produced a significant impairment in the phagocytic capacity of M1-activated microglia (2.7-fold lower than LPS, $p<0.001$; ns compared to untreated, Fig. 3c, f). Conversely, the addition of db-cyclic AMP alone significantly increased the phagocytic activity of the M1 phenotype (Fig. 3d) and additionally restored the deficits observed in the phagocytic properties conferred in IL-4 driven M2a microglia
(Fig. 3e, f), conferring a phagocytic activity significantly greater than that of LPS stimulation alone (1.4-fold and 1.3 -fold higher than LPS, $p<0.01$ and $p<0.05$, respectively; Fig. 3f). These results demonstrate that an important role of classically activated M1 microglia, phagocytosis and debris clearance, is retained in the converted M2a phenotype.

\section{Elevated levels of cyclic AMP combined with IL4 antagonizes the production of reactive oxygen and nitrogen species following microglial activation}

We examined whether M2a converted microglia, obtained through the combined exposure of M1 microglia to IL-4 and db-cyclic AMP, produced reactive oxygen (ROS) and/or nitrogen species (RNS), cytotoxic molecules that are associated with the M1 microglial cell phenotype. The ability of M1 microglia to produce RNS, measured by nitrite concentration, was detected based on the Griess reaction. Compared to untreated controls, LPS stimulation of BV2 microglia to the M1 form produced a significant increase in cell nitrite concentration (1.5-fold increase, $p<0.001$; Fig. 3g). Concurrent use of IL-4 with LPS significantly attenuated this increase (Fig. 3g). The employment of either db-cyclic AMP alone or M2a phenotypic conversion with the combination of IL-4 and db-cyclic AMP, further reduced nitrite concentrations to levels significantly lower than that of untreated controls (2.3-fold and 5.9-fold reduction compared to untreated, $p<0.001$ for both, respectively; Fig. 3g). Levels of ROS detected using DCFH-DA showed that LPS stimulation of BV2 microglia produced a significant increase in ROS production compared to untreated controls (1.4-fold increase, $p<0.05$; Fig. 3h). The concomitant use of IL-4 or db-cyclic AMP alone did not significantly reduce the increase in ROS produced by LPS stimulation. Only when db-cyclic AMP 

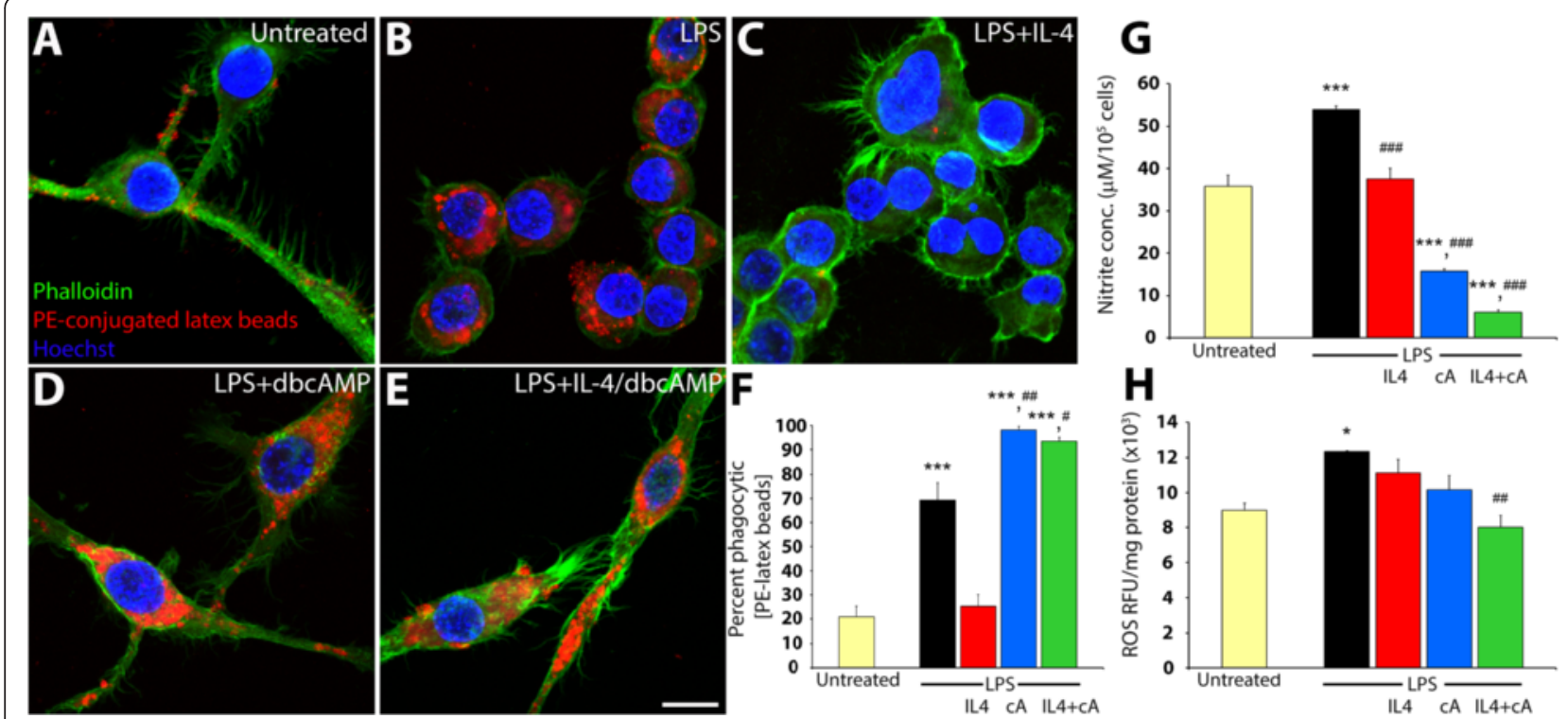

Fig. $3 \mathrm{M} 1$ to M2a phenotype conversion of microglia does not perturb their phagocytic function but does reduce the production of reactive species. a-e The phagocytic capacity of BV2 microglia was assayed by the ability of the cells to phagocytose phycoerythrin (PE)-conjugated latex beads (red) after treatment with LPS $(100 \mathrm{ng} / \mathrm{ml})$ in the presence or the absence of $\mathrm{IL}-4(10 \mathrm{ng} / \mathrm{ml})$ and db-cyclic AMP $(1 \mathrm{mM})$ added alone or simultaneously 15 min prior to activation and incubated for $24 \mathrm{~h}$ post-treatment. Co-staining for nuclei (Hoechst, blue) and the cytoplasm (phalloidin-488, green) was employed to demark cell morphology. Scale bar $=12 \mu \mathrm{m}$. f. Quantitative analysis of PE-bead uptake. $\mathbf{g}$, $\mathbf{h}$ Measurements of nitrite concentration (g) or reactive oxygen species (h) in cell lysates from untreated and treated groups were measured at $24 \mathrm{~h}$ post-stimulus with conditions inducing the pro-inflammatory or the anti-inflammatory state as mentioned above. Statistical significance indicated at ${ }^{* *} p<0.001$ or ${ }^{*} p<0.05$ versus untreated controls or ${ }^{\# \# \#} p<0.001$, \#\# $p<$ 0.01 , or ${ }^{\#} p<0.05$ versus LPS-only-treated controls. Results are shown from five independent culture replicates

and IL-4 were employed in combination to promote M2a phenotype conversion was the LPS-induced generation of ROS in microglia completely perturbed (1.5-fold decrease, $p<0.01$ compared to LPS stimulation; ns compared to untreated; Fig. $3 \mathrm{~h}$ ).

\section{Acute systemic co-administration of IL4 and cyclic AMP induces a state of alternative activation in microglia and macrophages after spinal cord injury}

To investigate whether M1 to M2a phenotypic conversion of microglia could be accomplished in vivo using cyclic AMP elevation and IL-4 supplementation, their concurrent delivery was investigated after contusive spinal cord injury (SCI) in two different experimental paradigms. First, in a rodent model, adult Lewis rats were subjected to injury and the co-administration of IL-4 and db-cyclic AMP systemically during the acute phase (within $15 \mathrm{~min}$ ) of SCI. Using the prototypical M2a marker Arginase-1, at 24 h after SCI an increase in Arginase-1 expression was identified in the total spinal cord tissue homogenates of the lesion site compared to naïve controls by immunoblotting (5.9-fold increase compared to naïve, $p<0.05$ Fig. 4a, b); indeed, an increase in both M1 and M2 microglia and macrophage populations has been reported previously in the acute phase of SCI [10]. The use of M1 to M2a phenotype conversion with db-cyclic AMP and IL-4 resulted in a significant enhancement of Arginase-1 production at $24 \mathrm{~h}$ after SCI (11.1-fold increase compared to naïve, $p$ $<0.01$ and $p<0.05$ versus SCI; Fig. 4a, b). Histological examination of tissue sections from the injury epicenter showed that, compared to SCI controls (Fig. 4c, d), the combination of db-cyclic AMP and IL-4 after SCI produced a marked reduction in numbers of $\mathrm{Iba1}^{+}$(Fig. 4e) and $\mathrm{ED} 1^{+}$(Fig. 4f) microglia and macrophages within the lesion site. Co-staining of $\mathrm{ED}^{+}$microglia or macrophages with Arginase- 1 revealed that at $24 \mathrm{~h}$ after SCI alone, only a small fraction of $\mathrm{ED}^{+}$cells were immunoreactive for Arginase-1 (Fig. 4d-f; white arrows). In contrast, when M2a phenotype conversion with db-cyclic AMP and IL-4 was used acutely after $\mathrm{SCI}$, almost all the $\mathrm{ED}^{+}{ }^{+}$microglia and macrophages were immunoreactive for Arginase-1 (Fig. 4h-j; white arrows), indicating that they were of the M2a phenotype. To assess the effects of M2 microglia and macrophage conversion on the pathological processes of injury, we assessed at $24 \mathrm{~h}$ the generation of total free ROS/RNS radicals within the lesion site by employing the fluorogenic probe dichlorodihydrofluorescin DiOxyQ (DCFH-DiOxyQ), which is a specific ROS/RNS probe [23]. Compared to SCI controls, the combination of dbcyclic AMP and IL-4 to induce M1 to M2a phenotypic conversion produced a significant reduction in the amount of free radicals generated within the injured spinal cord (57.6\% reduction, $p<0.05$; Fig. $4 \mathrm{k}$ ). 


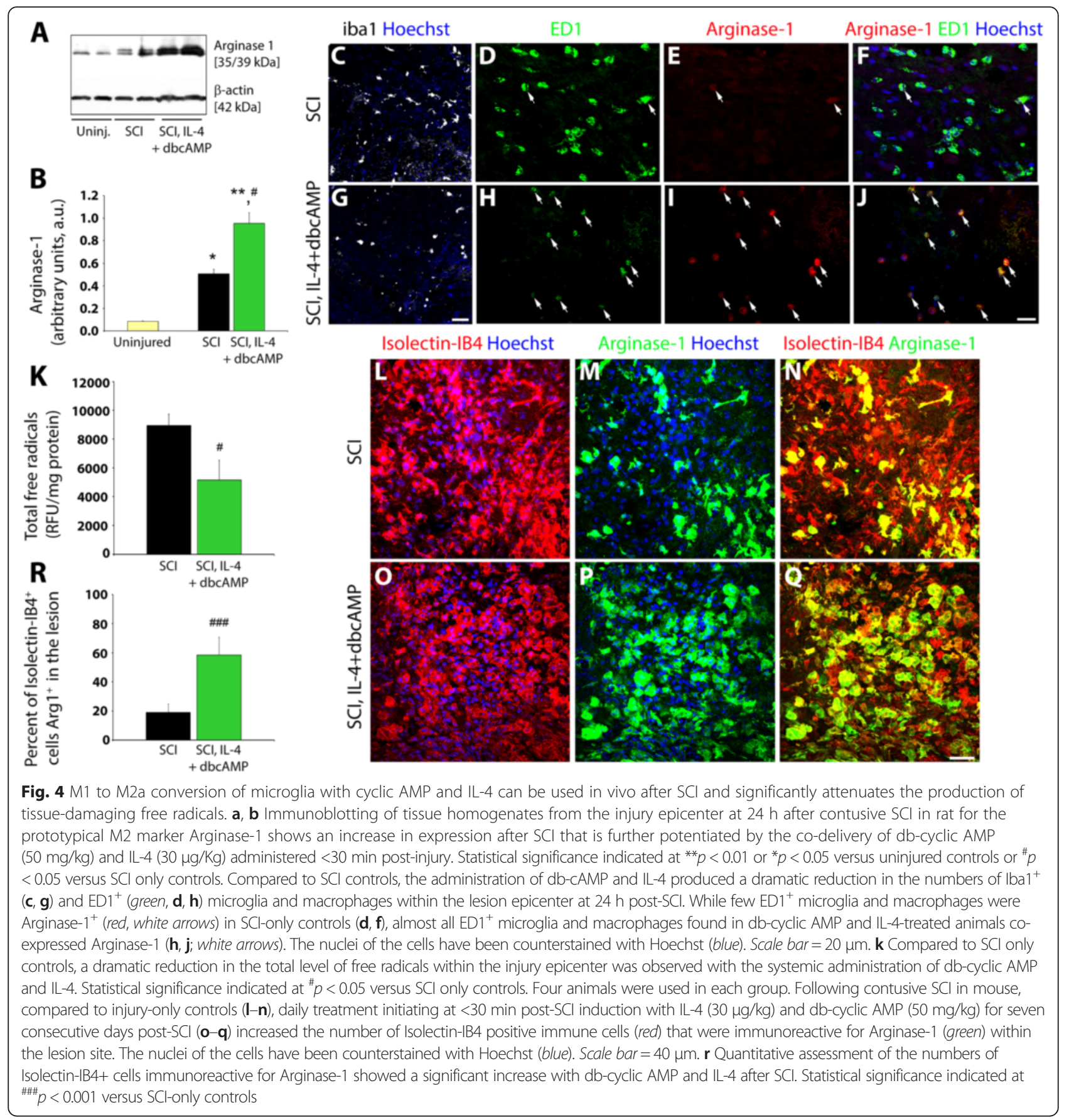

In subsequent work, we employed a murine model of moderate SCI using c57/Bl6 mice. These mice received either SCI alone or SCI with the co-administration of IL-4 and db-cyclic AMP acutely post-SCI and then daily for 7 days. Immunohistochemical analysis of spinal cord tissue at the lesion site at 7 days post-SCI showed that compared to SCI controls (Fig. 4l-n), a significant increase in the number of Isolectin-IB4. $4^{+}$microglia and macrophages immunoreactive for Arginase-1 occurred with the concurrent delivery of IL-4 and db-cyclic AMP (Fig. 4o-q). Arginase-1 immunoreactivity at the center of the lesion appeared largely restricted to Isolectin-IB4+ ${ }^{+}$cells. Quantitative assessment of the number of Isolectin-IB4+ ${ }^{+}$microglia and macrophages that co-stained with Arginase- 1 after SCI showed a significant increase with IL- 4 and db-cyclic AMP over SCI controls (3.0-fold increase; $p<0.001$; Fig. 4r).

\section{Discussion}

Trauma to the CNS produces an intense and chronic reaction to the injury by resident microglia and infiltrating 
macrophages that perplexingly when abrogated can lead to tissue preservation and a reduction in neurological deficits [24] or, when specifically enhanced, can produce repair [25]. Recent work has attributed these diverse activities to the heterogeneity of the microglia and macrophage responses to injury, with the presence of distinct immunophenotypical populations of these cells providing unique functional roles within the injury environment $[10,26]$. These phenotypes are broadly defined into two forms based upon gene profiling and proteomics, the classically activated M1 form and the alternatively activated M2 form, though it is clear that further work remains in the molecular identification of distinct microglial cell populations in vivo that possess different behavioral characteristics.

Though our understanding of the complexity of the microglia and macrophage responses following injury requires further experimentation to elucidate how these different populations alter the injury environment towards one of tissue damage or repair, it does appear based upon temporal population dynamics within the lesion milieu that the conversion of one phenotype to another is possible $[10,27,28]$ and that factors present within the injury microenvironment strongly regulate the microglia and macrophage phenotypic responses and function $[9,29,30]$. The dominating role of signals from the injury milieu in driving the M1 phenotype was recently demonstrated in studies by Kigerl et al., [10] where they showed that M2-converted macrophages, induced with IL-4, while retaining their phenotype when microinjected into the intact spinal cord, were converted into the M1 state when injected into the injured spinal cord. Indeed, while both M1 and M2 microglia and macrophages are present within the acutely injured spinal cord, the M2 response is short-lived, dissipating within 3-7 days after injury [10], pointing to an environment supportive of only the M1 phenotype [31].

With the injured CNS showing concurrently a dramatic and chronic reduction in levels of the important secondary messenger, cyclic AMP [32], and based upon the important role that cyclic AMP plays in maintaining both microglia and monocyte homeostasis to prevent M1 activation [15, 33], we hypothesized that the elevation of cyclic AMP would be a pre-requisite to pro-M2 anti-inflammatory cytokine stimulation for a persistent M2 phenotypical conversion from an M1 activated state.

As shown in the present report, the combination of cyclic AMP elevation and stimulation with IL-4, but neither agent alone at the doses employed, was able to convert LPS-activated, M1 microglia to an M2a phenotype as characterized by a number of specific M1 and M2a markers. Though further dose-finding studies with the prototypical M2 inducer IL-4 alone [34-36] may allow the determination of a concentration of IL- 4 that can achieve a significant induction of M2 markers in these cell culture systems, it is clear from this work that cyclic AMP provides a powerful synergism with IL-4 to induce M1 to M2a microglial conversion where individually these agents failed to at doses that are physiologically relevant. We and others have shown that cyclic AMPelevating agents, including adenylyl cyclase activators, phosphodiesterase inhibitors, and synthetic analogs can retard the classical (M1) activation of microglia or macrophages largely through antagonism of the master transcriptional regulator of inflammation, nuclear factor kappa B (NF-kB; $[11,33])$. In addition, cyclic AMP can also synergistically act with IL-4 to induce the expression of M2-regulated genes, such as Arginase-1, by activating the transcription factor CCAAT/enhancerbinding protein (C/EBP) [37]; thus, both of these mechanisms are likely important for the interplay of these signals in promoting persistent M1 to M2 conversion. According to recent sub-type classification of the M2 phenotype by immunocytochemical markers [9], the cyclic AMP and IL-4 converted microglia retain the M2a form; the substitution of IL-4 with other antiinflammatory cytokines, such as IL-10 or TGF- $\beta$, in conjunction with cyclic AMP failed to recapitulate this M1 to M2a microglia conversion (data not shown), while IL13 produced an analogous response to IL-4 with cyclic AMP (unpublished data). Functionally, the M2a conversion of M1 microglia with cyclic AMP and IL-4 significantly abated their production of pro-inflammatory cytokines (TNF- $\alpha$ ) and chemokines (IP-10) and oxidative molecules (ROS/RNS), while increasing their phagocytic function. The production of pro-inflammatory cytokines and oxidative metabolites are key effectors of M1 microglia in exacerbating tissue damage after CNS injury [2], while for M2 microglia, the down-regulation of these inflammatory factors coupled with enhanced phagocytosis and ECM remodeling are important for their reparative actions [4].

While in the current study, microglial cells treated with LPS did not exhibit Arginase-1 expression, other work has shown that LPS can increase Arginase-1 within microglia in vitro [38] and in vivo [39, 40]. However, in each of these cases, there was the presence of additional stimuli or environmental factors that could have altered the response of microglia to LPS. In work by Lisi and colleagues [38], LPS stimulation occurred in the presence of conditioned media from C6 Glioma cells, media containing growth factors, and cytokines that could have altered the response to LPS leading to Arginase-1 expression. Similarly, in a study by Zhang and co-workers [40], involving a model of Endotoxin-induced uveitis (EIU), the break-down of the blood-ocular barrier and the infiltration of leukocytes in the retina following administration of LPS may have led to indirect effects on the microglia from secreted factors from leukocytes [41] or other glial cells (Muller glia). Secreted factors from 
these cells could have altered the response of the microglia to LPS in their production of Arginase-1. This environmental difference could also be a reason for the observed induction of Arginase-1 in cortical microglia following intraparenchymal injection of LPS in the brain [40]. Examining how LPS-induced changes in Arginase1 expression by microglia may be altered by the presence of cytokines, growth factors, and other cell types would shed light on how the signaling pathways downstream of these factors are integrated in regulating microglial cell phenotype.

Through the use of PKA- and EPAC-selective cyclic AMP analogs, we demonstrated the importance of PKA, but not EPAC, in facilitating M1 to M2a microglia conversion in the presence of IL-4. Studies have shown that in LPS activated RAW 264.7 macrophages, the effects of cyclic AMP in reducing pro-inflammatory cytokine production, a hallmark of the M1 phenotype, is mediated through PKA [42]. Conversely, other work employing the same macrophage cell model has highlighted a role for Epac1-mediated Rap1/NFkB signaling in facilitating LPS-induced inflammatory responses [43]. While the use of EPAC and PKA agonists in the current study at concentrations previously shown to provide sufficient activation of the target in vitro [44] demonstrate the importance of PKA and not EPAC in M1 to M2a conversion and are in line with previous reports [43] that suggest the involvement of PKA and not EPAC as being the downstream mediator of M1 to M2 conversion; the use of multiple, higher doses of the EPAC agonists would be needed to completely rule out a role of EPAC in this process. Nevertheless, if demonstrated, it would indicate that selective PKA activation rather than broad cyclic AMP-elevating approaches will be required for

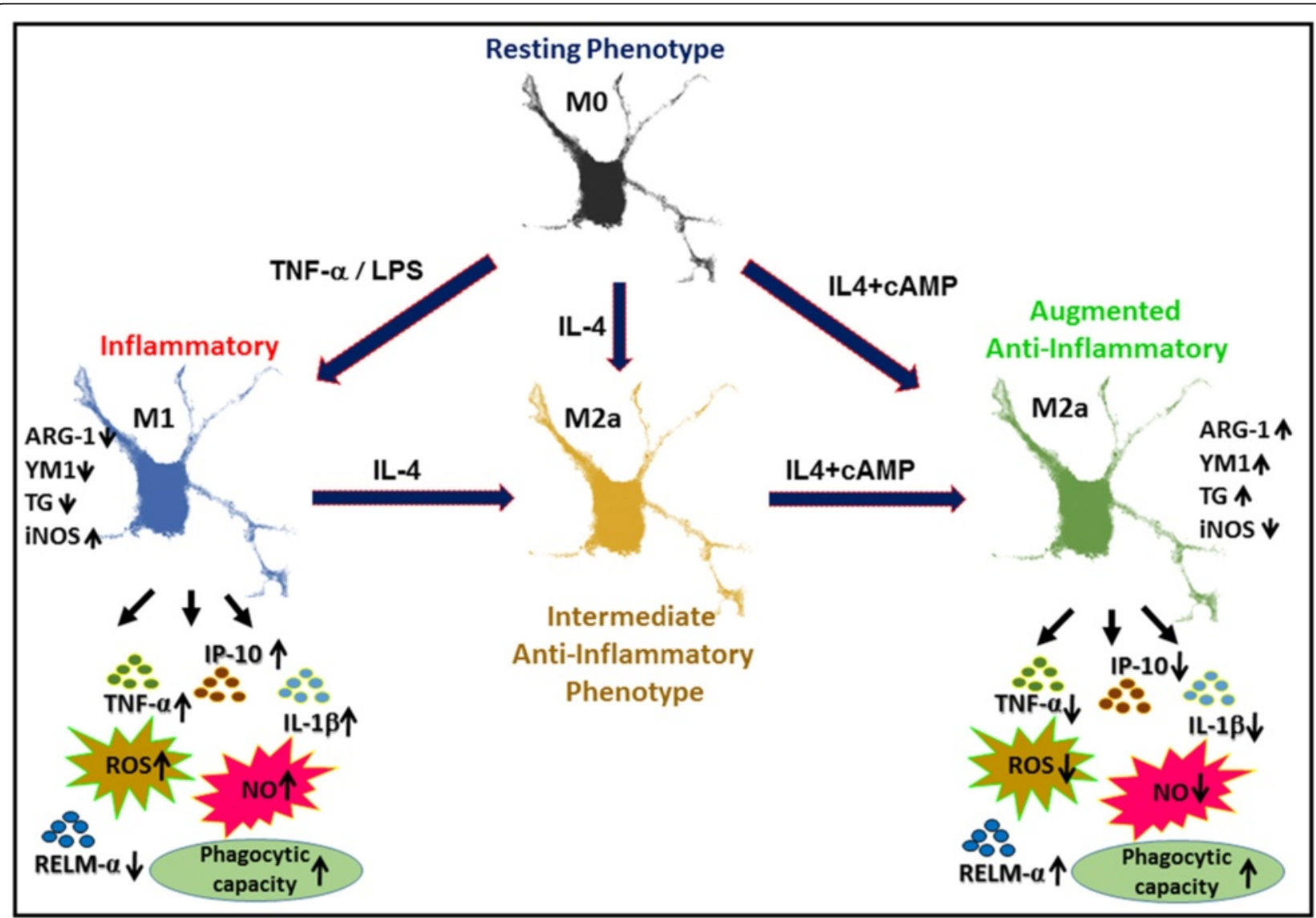

Fig. 5 Synergistic immuno-modulatory effects of cyclic AMP and IL-4 in the M1 to M2a phenotypic conversion of microglia. Subjecting resting (M0) microglia to a pro-inflammatory stimulus, TNF-a or LPS, drives the cells to a classically activated (M1) phenotype. The M1 form displays high expression of cytokines and chemokines, such as TNF- $a$ and IP-10, as well as iNOS and free radicles, ROS and RNS, that are key in cytotoxicity and tissue injury. In addition, the production of tissue reparative and remodeling enzymes, such as ARG-1, YM1, RELM-a, and TG, are suppressed. In contrast, the concurrent exposure of M0 or M1 microglia to IL-4 and cyclic AMP induces M0/M1 phenotype conversion to a robust reparative M2a form. The M2a phenotype exhibits strong expression of ARG-1, TG2, RELM- $a$, and YM1, an augmented phagocytic ability and the absence of pro-inflammatory cytokine and toxic reactive radical production that are associated with the $\mathrm{M} 1$ form. When $\mathrm{M0}$ - or M1-activated microglia are exposed to IL-4 alone, there is a transition to an intermediate M2a form. The M2a phenotype retains partial characteristics of M1 while acquiring some beneficial characteristics of the M2 phenotype, though exhibits impaired phagocytic function 
persistent M2 conversion to avoid putative antagonistic effects of EPAC activation.

Not only could M1 to M2a conversion be obtained phenotypically and functionally with combined cyclic AMP and IL-4 in vitro in the BV2 microglial cell line and primary microglia but also in vivo in models of SCI in rat and mouse. While only a small population of macrosialin-positive microglia and macrophages within the injured spinal cord at $24 \mathrm{~h}$ after SCI co-expressed the M2a marker Arginase-1, following the systemic administration of cyclic AMP and IL-4, almost all macrosialin immunoreactive microglia and macrophages were also Arginase-1 positive. In addition to promoting M1 to M2a conversion, this treatment also reduced the total numbers of macrosialin and iba-1 positive microglia and macrophages within the injured spinal cord. This reduction could be produced by an inhibitory effect of cyclic AMP on macrophage infiltration as observed previously with cyclic AMP-elevating approaches [20, 45]. A similar increase in Arginase-1 with cyclic AMP and IL-4 after SCI was observed also by immunoblotting, and the in vivo M1 to M2a conversion after SCI was accompanied by a significant reduction in the generation of ROS. In SCI mice treated with IL-4 and a cyclic AMP analog for 7 days after injury, a similar significant increase in Arginase $-1^{+}$M2a microglia and macrophages was observed with the combination of agents over injury-only controls, substantiating this approach as a means to promote M1 to M2a conversion in vivo after CNS injury as a putative therapeutic strategy for inducing tissue repair.

Although direct spinal tissue measurements of $\mathrm{db}$ cAMP and IL-4 were not performed in this study, following SCI, there is acute blood-spinal cord-barrier (BSCB) disruption to macromolecules for a least 1 week [46]. Disruption of the BSCB during the 7-day administration period of the agents would thus permit systemically administered IL4 and db-cAMP to readily reach spinal tissue at the lesion site. Should longer administration windows be required for achieving an effective treatment with this approach following SCI, the examination of spinal cord penetrance would be important to ensure that adequate levels were present or an alternative, more direct route for delivery, or small molecule mimetics/activators of these pathways would be needed.

\section{Conclusions}

These studies identify a novel method for M1 to M2a conversion that could hold great clinical promise as a therapeutic for CNS injury and disease in altering the microglia and macrophage responses from one of tissue injury to one of repair. Undertaking further in vivo work to optimize dosing of the agents and to determine the persistence of phenotypic conversion after CNS injury would pave the way for studies to examine the effectiveness of this new approach to provide anatomical protection, neurorepair, and functional benefit. It is also clear that further characterization of the synergistic mechanisms involved in M1 to M2 conversion and improved characterization of the relationship between the molecular profile of distinct microglia phenotypes and their behavioral responses is needed. As summarized in Fig. 5, the current work highlights for the first time the importance of cyclic AMP-PKA signaling in both antagonizing M1 microglia activation and inducing the M2a state in conjunction with anti-inflammatory cytokines, such as IL-4, to promote M1 to M2a phenotypical conversion.

\section{Competing interests}

The authors declare that they have no competing interests.

\section{Authors' contributions}

MG designed the experiments, contributed to biochemical, histological, and imaging aspects of the experiments as well as was involved in data acquisition, analysis, and interpretation and writing the manuscript. YX contributed to all surgical and histological aspects of the study including the analysis of tissue specimens. DDP designed the experiments, provided critical interpretation of the data, and was involved in the writing of the manuscript. All authors have read and approved the final manuscript.

\section{Acknowledgements}

We would like to thank Ms. Khine Wai for her technical assistance with cell culture and biochemistry as well as Dr. Alexander Marcillo and The Miami Project Animal Core Facility staff for their help with the animal work. Dr. Melissa Carballosa-Gautam of The Miami Project Imaging Core is thanked for her assistance with microscopy. We gratefully acknowledge funding support from The Paralyzed Veterans' of America, NIH NINDS award NS077142, and The Miami Project to Cure Paralysis.

\section{Author details}

${ }^{1}$ The Miami Project to Cure Paralysis, University of Miami Miller School of Medicine, Miami, FL 33136, USA. ²Department of Neurological Surgery, University of Miami Miller School of Medicine, Miami, FL 33136, USA. ${ }^{3}$ The Neuroscience Program, University of Miami Miller School of Medicine, Miami, FL 33136, USA. ${ }^{4}$ The Interdisciplinary Stem Cell Institute, University of Miami Miller School of Medicine, Miami, FL 33136, USA.

Received: 13 October 2015 Accepted: 17 December 2015

Published online: 13 January 2016

\section{References}

1. Pineau I, Lacroix S. Proinflammatory cytokine synthesis in the injured mouse spinal cord: multiphasic expression pattern and identification of the cell types involved. J Comp Neurol. 2007;500:267-85.

2. Banati RB, Gehrmann J, Schubert P, Kreutzberg GW. Cytotoxicity of microglia. Glia. 1993;7:111-8.

3. Mantovani A, Biswas SK, Galdiero MR, Sica A, Locati M. Macrophage plasticity and polarization in tissue repair and remodelling. J Pathol. 2013; 229:176-85.

4. Gordon S. Alternative activation of macrophages. Nat Rev Immunol. 2003;3: 23-35.

5. Streit WJ, Graeber MB, Kreutzberg GW. Functional plasticity of microglia: a review. Glia. 1988;1:301-7.

6. Benoit M, Desnues B, Mege JL. Macrophage polarization in bacterial infections. J Immunol. 2008;181:3733-9.

7. Popovich PG, Guan Z, McGaughy V, Fisher L, Hickey WF, Basso DM. The neuropathological and behavioral consequences of intraspinal microglial/ macrophage activation. J Neuropathol Exp Neurol. 2002;61:623-33.

8. Chhor V, Le Charpentier T, Lebon S, Ore MV, Celador IL, Josserand J, et al. Characterization of phenotype markers and neuronotoxic potential of polarised primary microglia in vitro. Brain Behav Immun. 2013;32:70-85. 
9. David S, Kroner A. Repertoire of microglial and macrophage responses after spinal cord injury. Nat Rev Neurosci. 2011;12:388-99.

10. Kigerl KA, Gensel JC, Ankeny DP, Alexander JK, Donnelly DJ, Popovich PG. Identification of two distinct macrophage subsets with divergent effects causing either neurotoxicity or regeneration in the injured mouse spinal cord. J Neurosci. 2009;29:13435-44.

11. Gerlo S, Kooijman R, Beck IM, Kolmus K, Spooren A, Haegeman G. Cyclic AMP: a selective modulator of NF-kappaB action. Cell Mol Life Sci. 2011;68: 3823-41.

12. Hayden MS, Ghosh S. Regulation of NF-kappaB by TNF family cytokines. Semin Immunol. 2014;26:253-66.

13. Blasi E, Barluzzi R, Bocchini V, Mazzolla R, Bistoni F. Immortalization of murine microglial cells by a v-raf/v-myc carrying retrovirus. J Neuroimmunol. 1990;27:229-37.

14. Henn A, Lund S, Hedtjarn M, Schrattenholz A, Porzgen P, Leist M. The suitability of BV2 cells as alternative model system for primary microglia cultures or for animal experiments examining brain inflammation. ALTEX. 2009;26:83-94.

15. Ghosh M, Garcia-Castillo D, Aguirre V, Golshani R, Atkins CM, Bramlett HM, et al. Proinflammatory cytokine regulation of cyclic AMP-phosphodiesterase 4 signaling in microglia in vitro and following CNS injury. Glia. 2012;60:1839-59.

16. Gruner JA. A monitored contusion model of spinal cord injury in the rat. J Neurotrauma. 1992:9:123-6. discussion 126-128.

17. Ghosh M, Tuesta LM, Puentes R, Patel S, Melendez K, El Maarouf A, et al. Extensive cell migration, axon regeneration, and improved function with polysialic acid-modified Schwann cells after spinal cord injury. Glia. 2012; 60:979-92.

18. Nishi RA, Liu H, Chu Y, Hamamura M, Su MY, Nalcioglu O, et al. Behavioral, histological, and ex vivo magnetic resonance imaging assessment of graded contusion spinal cord injury in mice. J Neurotrauma. 2007;24:674-89.

19. Patel V, Joseph G, Patel A, Patel S, Bustin D, Mawson D, et al. Suspension matrices for improved Schwann-cell survival after implantation into the injured rat spinal cord. J Neurotrauma. 2010;27:789-801.

20. Schaal SM, Garg MS, Ghosh M, Lovera L, Lopez M, Patel M, et al. The therapeutic profile of rolipram, PDE target and mechanism of action as a neuroprotectant following spinal cord injury. PLoS One. 2012;7, e43634.

21. Barakat DJ, Gaglani SM, Neravetla SR, Sanchez AR, Andrade CM, Pressman Y, et al. Survival, integration, and axon growth support of glia transplanted into the chronically contused spinal cord. Cell Transplant. 2005;14:225-40.

22. Cheng X, Ji Z, Tsalkova T, Mei F. Epac and PKA: a tale of two intracellular cAMP receptors. Acta Biochim Biophys Sin (Shanghai). 2008;40:651-62.

23. Xu Y, Duan C, Kuang Z, Hao Y, Jeffries JL, Lau GW. Pseudomonas aeruginosa pyocyanin activates NRF2-ARE-mediated transcriptional response via the ROS-EGFR-PI3K-AKT/MEK-ERK MAP kinase signaling in pulmonary epithelial cells. PLoS One. 2013;8, e72528.

24. Stirling DP, Yong VW. Dynamics of the inflammatory response after murine spinal cord injury revealed by flow cytometry. J Neurosci Res. 2008:86:1944-58.

25. Rabchevsky AG, Streit WJ. Grafting of cultured microglial cells into the lesioned spinal cord of adult rats enhances neurite outgrowth. J Neurosci Res. 1997:47:34-48.

26. London A, Cohen M, Schwartz M. Microglia and monocyte-derived macrophages: functionally distinct populations that act in concert in CNS plasticity and repair. Front Cell Neurosci. 2013;7:34.

27. Imagama T, Ogino K, Takemoto K, Kato Y, Kataoka H, Suzuki H, et al. Regulation of nitric oxide generation by up-regulated arginase I in rat spinal cord injury. J Clin Biochem Nutr. 2012;51:68-75.

28. Shin T, Ahn M, Moon C, Kim S, Sim KB. Alternatively activated macrophages in spinal cord injury and remission: another mechanism for repair? Mol Neurobiol. 2013:47:1011-9.

29. Kroner A, Greenhalgh AD, Zarruk JG, Passos Dos Santos R, Gaestel M, David S. TNF and increased intracellular iron alter macrophage polarization to a detrimental M1 phenotype in the injured spinal cord. Neuron. 2014;83:1098-116.

30. Wang X, Cao K, Sun X, Chen Y, Duan Z, Sun L, et al. Macrophages in spinal cord injury: phenotypic and functional change from exposure to myelin debris. Glia. 2015;63:635-51.

31. Huang W, Vodovotz Y, Kusturiss MB, Barclay D, Greenwald K, Boninger ML, et al. Identification of distinct monocyte phenotypes and correlation with circulating cytokine profiles in acute response to spinal cord injury: a pilot study. PM R. 2014;6:332-41.
32. Pearse DD, Pereira FC, Marcillo AE, Bates ML, Berrocal YA, Filbin MT, et al. cAMP and Schwann cells promote axonal growth and functional recovery after spinal cord injury. Nat Med. 2004;10:610-6.

33. Ghosh M, Aguirre V, Wai K, Felfly H, Dietrich WD, Pearse DD. The interplay between cyclic AMP, MAPK, and NF-kappaB pathways in response to proinflammatory signals in microglia. BioMed Research International. 2015; 2015:308461.

34. Fenn AM, Hall JC, Gensel JC, Popovich PG, Godbout JP. IL-4 signaling drives a unique arginase+//L-1 beta+ microglia phenotype and recruits macrophages to the inflammatory CNS: consequences of age-related deficits in IL-4Ralpha after traumatic spinal cord injury. J Neurosci. 2014; 34:8904-17.

35. Pepe G, Calderazzi G, De Maglie M, Villa AM, Vegeto E. Heterogeneous induction of microglia M2a phenotype by central administration of interleukin-4. J Neuroinflammation. 2014;11:211.

36. Latta CH, Sudduth TL, Weekman EM, Brothers HM, Abner EL, Popa GJ, et al. Determining the role of IL-4 induced neuroinflammation in microglial activity and amyloid-beta using BV2 microglial cells and APP/ PS1 transgenic mice. J Neuroinflammation. 2015;12:41.

37. Sheldon KE, Shandilya H, Kepka-Lenhart D, Poljakovic M, Ghosh A, Morris Jr SM. Shaping the murine macrophage phenotype: IL-4 and cyclic AMP synergistically activate the arginase I promoter. J Immunol. 2013;191:2290-8.

38. Lisi L, Laudati E, Navarra P, Dello Russo C. The mTOR kinase inhibitors polarize glioma-activated microglia to express a M1 phenotype. J Neuroinflammation. 2014;11:125.

39. Zhang W, Baban B, Rojas M, Tofigh S, Virmani SK, Patel C, et al. Arginase activity mediates retinal inflammation in endotoxin-induced uveitis. Am J Pathol. 2009;175:891-902.

40. Lee DC, Rizer J, Selenica ML, Reid P, Kraft C, Johnson A, et al. LPS- induced inflammation exacerbates phospho-tau pathology in rTg4510 mice. J Neuroinflammation. 2010;7:56.

41. Gadani SP, Cronk JC, Norris GT, Kipnis J. IL-4 in the brain: a cytokine to remember. J Immunol. 2012;189:4213-9.

42. Wall EA, Zavzavadjian JR, Chang MS, Randhawa B, Zhu X, Hsueh RC, et al. Suppression of LPS-induced TNF-alpha production in macrophages by CAMP is mediated by PKA-AKAP95-p105. Sci Signal. 2009;2:ra28.

43. Moon EY, Pyo S. Lipopolysaccharide stimulates Epac1-mediated Rap1/NFkappaB pathway in RAW 264.7 murine macrophages. Immunol Lett. 2007; 110:121-5.

44. Misra UK, Pizzo SV. Upregulation of mTORC2 activation by the selective agonist of EPAC, 8-CPT-2Me-CAMP, in prostate cancer cells: assembly of a multiprotein signaling complex. J Cell Biochem. 2012;113:1488-500.

45. Bao F, Fleming JC, Golshani R, Pearse DD, Kasabov L, Brown A, et al. A selective phosphodiesterase-4 inhibitor reduces leukocyte infiltration, oxidative processes, and tissue damage after spinal cord injury. J Neurotrauma. 2011;28:1035-49.

46. Figley SA, Khosravi R, Legasto JM, Tseng YF, Fehlings MG. Characterization of vascular disruption and blood-spinal cord barrier permeability following traumatic spinal cord injury. J Neurotrauma. 2014;31:541-52.

\section{Submit your next manuscript to BioMed Central and we will help you at every step:}

- We accept pre-submission inquiries

- Our selector tool helps you to find the most relevant journal

- We provide round the clock customer support

- Convenient online submission

- Thorough peer review

- Inclusion in PubMed and all major indexing services

- Maximum visibility for your research

Submit your manuscript at www.biomedcentral.com/submit 